\title{
Aplicação estatística multivariada para a avaliação físico-química na qualidade da água subterrânea na cidade de Parauapebas (Sudeste do Estado do Pará)
}

Amostras de águas subterrâneas foram coletadas em 20 locais de amostragem distribuídos em nove bairros da Cidade de Parauapebas (Estado do Pará, Brasil), com o objetivo de avaliar a qualidade das águas subterrâneas captadas em poços tubulares potencialmente utilizados para consumo humano. Para todas as amostras de águas subterrâneas foram analisados nove parâmetros físico-químicos, tais como temperatura (T), potencial hidrogeniônico ( $\mathrm{pH}$ ), condutividade elétrica (CE), turbidez (TRB), cor, teor de cloreto (Cl-) e sólidos totais dissolvidos (STD). A partir desses resultados e para melhor entender o comportamento físico-químico dessas águas, métodos multivariados, tais como a análise de componentes principais (PCA) e análise de agrupamento hierárquico (HCA) foram usadas para proceder a uma avaliação mais completa dos dados originais. Os parâmetros físico-químicos estudados mostraram que nas águas subterrâneas coletadas em poços tubulares no bairro Da Paz foram encontrados os valores mais elevados de sólidos totais dissolvidos $(175,0$ a $271,8 \mathrm{mg} \mathrm{L}-1)$, condutividade elétrica $(136,3$ a $216,7 \mu \mathrm{S} \mathrm{cm}-1)$ e cor $(5,0$ a $15,0 \mathrm{uH})$, indicando a influência de atividades antropogênicas de contaminação na região, principalmente relacionado a descarga de efluentes domésticos. Além disso, a PCA explicou $75,5 \%$ da variância total dos dados, enquanto a HCA confirmou as correlações encontradas na PCA, possibilitando avaliar o grau de similaridade entre as amostras e identificar os bairros mais propensos à contaminação de suas águas subterrâneas.

Palavras-chave: Água Subterrânea; Parâmetros Físico-Químicos; Análise Multivariada.

\section{Multivariate statistical application for the evaluation physicochemical in groudwater quality from the city of Parauapebas (Southeast of State Pará)}

Groundwater were sampled at 20 sampling locations of nine districts of Parauapebas City (Pará State, Brazil), with objective of evaluate the quality of groundwater sampled in tubular wells potentially used for human consumption. For all groundwater samples were analyzed nine physicochemical parameters, such as temperature $(\mathrm{T})$, hydrogen potential $(\mathrm{pH})$, electrical conductivity (EC), turbidity (TRB), color, chloride content (CI-) and total dissolved solids (TDS). From these results and to better understand the physicochemical behavior of these waters, multivariate methods such as principal component analysis (PCA) and hierarchical cluster analysis (HCA) were used to conduct a more thorough evaluation of the original data. The physicochemical parameters studied showed that the groundwater sampled in tubular wells from district of $\mathrm{Da}$ Paz, had the highest total dissolved solids (175.0 to $271.8 \mathrm{mg} \mathrm{L}-1$ ), electrical conductivity (136.3 at $216.7 \mathrm{\mu S} \mathrm{cm}-1$ ) and color (5.0 to $15.0 \mathrm{uH}$ ) values, indicating the influence of anthropogenic activities contamination in the region, mainly related to the discharge of domestic effluents. Furthermore, PCA explained $75.5 \%$ of the total variance, while the HCA confirmed the correlations found in PCA, allowing evaluating the degree of similarity between samples and identifying the districts most prone to groundwater contamination.

Keywords: Groundwater; Physicochemical Parameters; Multivariate Analysis.

Topic: Engenharia de Recursos Hídricos

Reviewed anonymously in the process of blind peer.

Henrique Sousa Chaves

Universidade Federal Rural da Amazônia,

Brasil

http://lattes.cnpq.br/2142593099019750

hs.chaves123@gmail.com

Denilton Galvão de Morais

Universidade Federal Rural da Amazônia,

Brasil

http://lattes.cnpq.br/5648851496617628 denilton475@gmail.com

Heronides Adonias Dantas Filho (iD Universidade Federal do Pará, Brasil http://lattes.cnpq.br/7383551694064339 http://orcid.org/0000-0001-5698-5441 herondantas@gmail.com

\section{Kelly das Graças Fernandes Dantas (iD) Universidade Federal do Pará, Brasil http://lattes.cnpq.br/72277777727553334 http://orcid.org/0000-0002-5424-2908 kdgfernandes@ufpa.br \\ Antônio Thiago Madeira Beirão (iD) Universidade Federal Rural da Amazônia, Brasil \\ http://lattes.cnpq.br/89490068888604428 http://orcid.org/0000-0003-1366-5995 thiago.ppgee.ufpa@gmail.com \\ Katiane Pereira da Silva \\ Universidade Federal Rural da Amazônia, Brasil \\ http://lattes.cnpq.br/3810443896855581 http://orcid.org/0000-0002-1697-6804 katianeperei@gmail.com}

Received: 05/08/2020

Approved: 26/09/2020

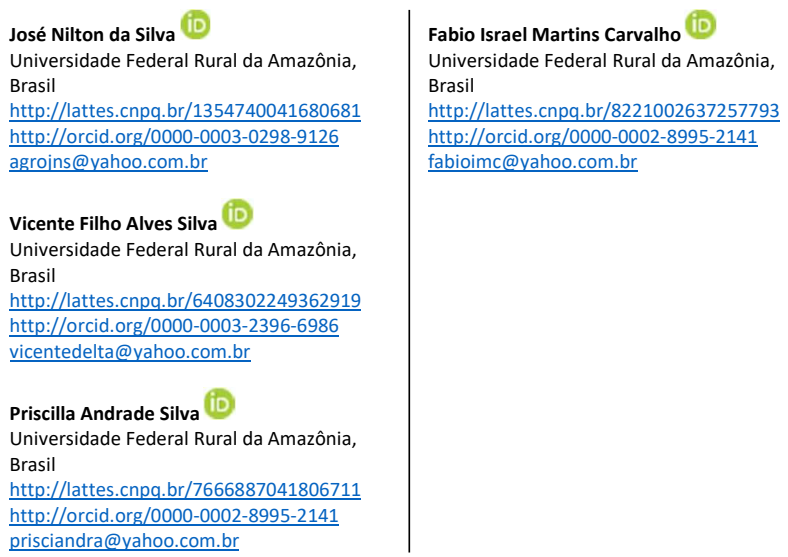

Referencing this:

CHAVES, H. S.; MORAIS, D. G.; DANTAS FILHO, H. A.; DANTAS, K. G. F.; BEIRAO, A. T. M.; SILVA, K. P.; SILVA, J. N.; SILVA, V. F. A.; SILVA, P. A.; CARVALHO, F. I. M.. Aplicação estatística multivariada para a avaliação físico-química na qualidade da água subterrânea na cidade de Parauapebas (Sudeste do Estado do Pará). Revista Ibero Americana de Ciências Ambientais, v.11, n.5, p.261-272, 2020. DOI: http://doi.org/10.6008/CBPC2179-6858.2020.005.0025 
Aplicação estatística multivariada para a avaliação físico-química na qualidade da água subterrânea na cidade de Parauapebas (Sudeste do Estado do Pará)

CHAVES, H. S.; MORAIS, D. G.; DANTAS FILHO, H. A.; DANTAS, K. G. F.; BEIRAO, A. T. M.; SILVA, K. P.; SILVA, J. N.; SILVA, V. F. A.; SILVA, P. A.; CARVALHO, F. I. M.

\section{INTRODUÇÃO}

O comprometimento da qualidade das águas subterrâneas se intensifica nos grandes centros urbanos, principalmente pelo uso e ocupação do solo, gerando efluentes diversos que retornam para os corpos hídricos, interferindo em sua qualidade, e em menor intensidade pela sazonalidade (AZEVEDO, 2006; CAMPANHA et al., 2010). Por isso, o monitoramento de águas subterrâneas empregando análises químicas é uma medida importante para avaliar a sua qualidade, servindo de indicativos para identificação de possíveis fontes de contaminação, que podem alterar significativamente as propriedades químicas da água, comprometendo o equilíbrio geral do sistema, causando prejuízos econômicos e inviabilizando seu consumo (REBOUÇAS et al., 2006).

Segundo Rebouças et al. (2006), as águas subterrâneas vêm perdendo em qualidade devido à sua contaminação por fossas sépticas, aterros sanitários, contaminação por pesticidas, fertilizantes, intrusão salina, fraturas em oleodutos, poços abandonados ou mal selados, resíduos industriais, depósitos subterrâneos de produtos químicos e vazamento tanques de armazenamento subterrâneo de combustíveis, etc. A contaminação de aquíferos por diversas fonte de contaminação antropogênicas e/ou naturais vem sendo uma preocupação mundial e tem sido também muito discutida no Brasil (DÓREA et al., 2007; FORTE et al., 2007).

Na região urbana da Cidade de Parauapebas, Sudeste do Estado do Pará, uma grande quantidade de condomínios residências e domicílios particulares utilizam para abastecimento, poços tubulares e escavados do tipo "Amazonas" como fonte de captação de água de mananciais subterrâneos, proveniente de corpo hidrogeológico (CARVALHO et al., 2019). Além disso, estes poços são perfurados, na maioria das vezes, sem levar em consideração os aspectos hidrogeológicos desse manancial e as proximidades de fossas (sépticas ou rudimentares) e esgotos, nem são sempre construídos obedecendo a critérios técnicos recomendados pela Associação Brasileira de Normas Técnicas (ABNT), apresentando grande vulnerabilidade à contaminação (CARVALHO et al., 2015, CARVALHO et al., 2020).

No Brasil, existem duas legislações vigentes para avaliação da qualidade da água, a Portaria № 2914/2011 do Ministério da Saúde trata dos procedimentos de controle e de vigilância da qualidade da água subterrânea e superficial para consumo humano, relativas aos padrões de potabilidade (BRASIL, 2011) e, a Resolução № 396/2008 do Conselho Nacional do Meio Ambiente estabelece a classificação das águas subterrâneas e as diretrizes ambientais para o seu enquadramento, bem como algumas definições relacionadas à suas características e os padrões estabelecidos conforme o seu uso (CONAMA, 2008).

Nos últimos anos a aplicação de métodos estatísticos multivariados como análise de componentes principais (PCA) e a análise hierárquica de agrupamentos (HCA) tem sido utilizado com frequência em diversos estudos reportados na literatura como ferramenta quimiométrica útil para extrair um maior número de informações obtidas através de análises de parâmetros físico-químicos, microbiológicos e elementos metálicos em amostras de águas superficiais, subterrâneas, chuva e minerais (CARVALHO et al., 2015).

Desta forma, o objetivo deste estudo foi avaliar a qualidade das águas subterrâneas captadas em 
poços tubulares potencialmente utilizados para consumo humano pela população de nove bairros localizados na Cidade de Parauapebas (Sudeste do Estado do Pará) a partir da determinação de parâmetros físicoquímicos das mesmas. Além disso, a análise multivariada (PCA e HCA) foi usada para uma avaliação mais completa dos dados originais, possibilitando uma extração máxima de informações para melhor interpretar e evidenciar possíveis correlações entre amostras e variáveis.

\section{MATERIAIS E MÉTODOS}

\section{Descrição da área de estudo}

O estudo foi conduzido na área urbana da Cidade de Parauapebas/PA, localizada ao Norte do Brasil e Sudeste do Estado do Pará, de acordo com as seguintes coordenadas: $6.0675^{\circ} \mathrm{S}$ e $49.9022^{\circ} \mathrm{W}$, como se pode observar na Figura 1, a qual ilustra os limites territoriais do município, onde a área urbana é marcada pelo contraste mais escuro.

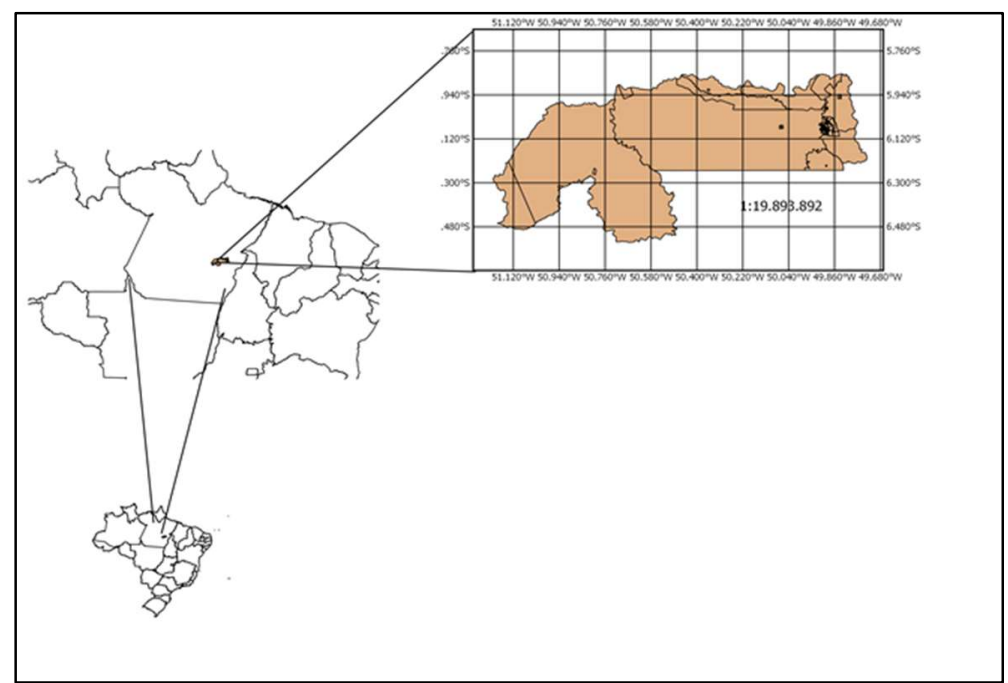

Figura 1: Delimitação territorial do município de Parauapebas.

Inicialmente foi feito um estudo da área, em consonância com a elaboração de um mapa vetorial através do programa Quantum Gis em sua versão 2.18 e com base de dados, referentes as malhas municipais do ano de 2010 (último senso demográfico), fornecidas por meio do site do Instituto Brasileiro de Geografia e Estatística (IBGE). Como os dados obtidos não condiziam com a atualidade, foi elaborado um novo mapa com auxílio do Google Maps e da ferramenta QuickMapServices, presente no software de mapeamento utilizado. Vale ressaltar que os mapas de bairros podem não representar a realidade de forma verossímil, isso, em função da ausência de dados referentes as fronteiras de alguns desses.

Observa-se na Figura 2 a malha urbana do município seguida de um mapa de ampliado para os bairros onde foram realizadas as coletas. A zona ampliada apresenta, quase em sua totalidade, um complexo de bairros centrais, com elevada concentração habitacional e de estabelecimentos comerciais, são eles: Da Paz,

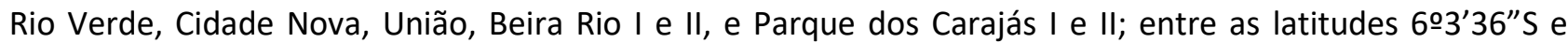
65'24"S, e no intervalo das longitudes 4954'36"W e 4952'12"W. 


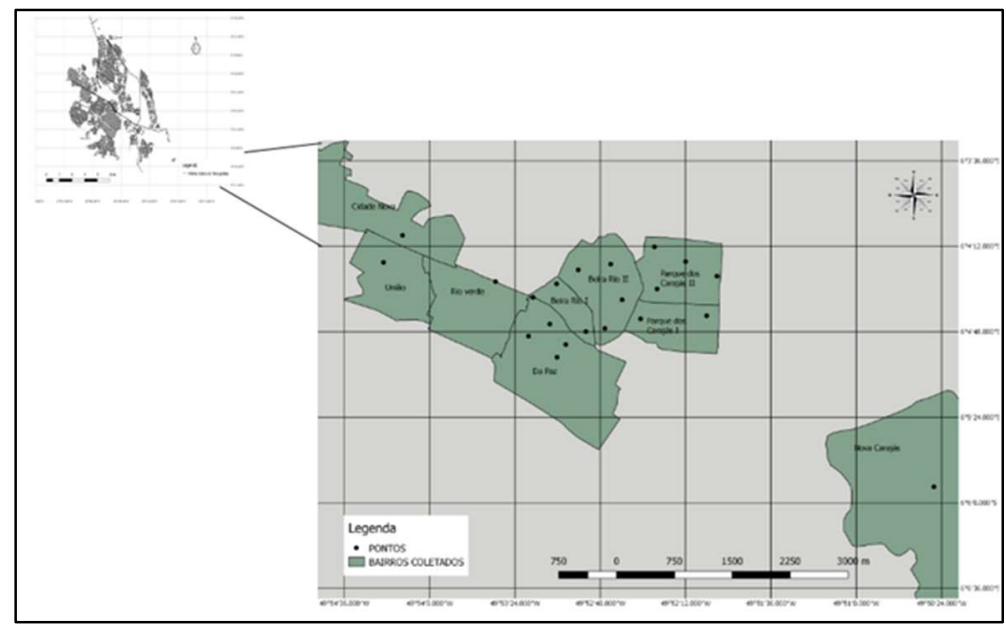

Figura 2: Malha municipal de Parauapebas e ampliação dos respectivos pontos de coleta distribuídos nos nove bairros em estudo.

O maior número de coletas realizadas foi, especialmente nos bairros da Paz, Beira Rio I e II e Parque dos Carajás I e II, devido à predominância de poços tubulares de captação de água subterrânea. A exceção foi o bairro Nova Carajás, que se encontra afastado da região central do município, no qual foi definido apenas um ponto de coleta. Nos bairros União e Rio Verde, por outro lado, as residências consultadas apresentavam, predominantemente, abastecimento da rede pública de água, reduzindo significativamente o número de amostras coletadas nos bairros citados.

Os bairros em estudo estão localizados próximos às micro-bacias hidrográficas que cortam a Cidade de Parauapebas, sendo um aspecto hidrográfico marcante, e por consequência da expansão e desenvolvimento urbano, foram transformados em esgotos a céu aberto, recebendo efluentes domésticos e industriais sem que houvesse algum tratamento prévio destes resíduos (CARVALHO et al., 2015). Todos os pontos de coleta foram georreferenciados utilizando um aparelho de localização (global positioning system, modelo Garmin Map 76).

As coletas foram realizadas em residências e condomínios residências horizontais e verticais, que fazem uso de água subterrânea proveniente do lençol freático. Os pontos de coleta, bairros, código de amostras e suas coordenadas geográficas estão descritos de acordo com a Tabela 1.

Tabela 1: Coordenadas geográficas dos 20 pontos coletados nos nove bairros da cidade de Parauapebas-PA.

\begin{tabular}{|c|c|c|c|}
\hline Pontos de coleta & Bairro & Código das amostras & Coordenadas geográficas \\
\hline 1 & Rio Verde & RV1 & 604'27,12"S e 49 $53^{\prime} 32,14^{\prime \prime} \mathrm{W}$ \\
\hline 2 & Da Paz & DP1 & 604'32,97"S e 4953'19,85"W \\
\hline 3 & Da Paz & DP2 & 604'49,11"S e 4953'18,39"W \\
\hline 4 & Da Paz & DP3 & 64'44,62"S e 4953'5,87"W \\
\hline 5 & Da Paz & DP4 & 604'52,4"S e 4953'2,35"W \\
\hline 6 & Da Paz & DP5 & 604'56,2"S e 4953'6,24"W \\
\hline 7 & União & UN1 & 604'16,64"S e 4954'23,84"W W \\
\hline 8 & Cidade Nova & CN1 & 604'4,81"S e 4954'14,04"W W \\
\hline 9 & Nova Carajás & NC1 & 605'52,81"S e 4950'27,41"W W \\
\hline 10 & Beira Rio I & $\mathrm{BRI}(1)$ & 604'27,12"S e $49^{\circ} 53^{\prime} 32,14^{\prime \prime} \mathrm{W}$ \\
\hline 11 & Beira Rio I & $\mathrm{BRI}(2)$ & 604'32,97"S e 4953'19,85"W W \\
\hline 12 & Beira Rio II & BRII(1) & 604'49,11"S e 4953'18,39"W W \\
\hline 13 & Beira Rio II & BRII(2) & 604'44,62"S e 4953'5,87"W W \\
\hline 14 & Beira Rio II & BRII(3) & $604^{\prime} 52,4^{\prime \prime}$ S e 4953'2,35"W \\
\hline
\end{tabular}


Aplicação estatística multivariada para a avaliação físico-química na qualidade da água subterrânea na cidade de Parauapebas (Sudeste do Estado do Pará)

CHAVES, H. S.; MORAIS, D. G.; DANTAS FILHO, H. A.; DANTAS, K. G. F.; BEIRAO, A. T. M.; SILVA, K. P.; SILVA, J. N.; SILVA, V. F. A.; SILVA, P. A.; CARVALHO, F. I. M.

\begin{tabular}{|c|c|c|c|}
\hline 15 & Beira Rio II & BRII(4) & 604'56,2"S e 4953'6,24"W \\
\hline 16 & Parque dos Carajás I & $\mathrm{PCl}(1)$ & 604'16,64"S e 4954'23,84"W W \\
\hline 17 & Parque dos Carajás I & $\mathrm{PCl}(2)$ & 604'4,81"S e 4954'14,04"W W \\
\hline 18 & Parque dos Carajás II & $\mathrm{PCII}(1)$ & 65'52,81"S e 4950'27,41"W W \\
\hline 19 & Parque dos Carajás II & $\mathrm{PCII}(2)$ & 604'4,81"S e 4954'14,04"W \\
\hline 20 & Parque dos Carajás II & $\mathrm{PCII}(3)$ & 65'52,81"S e 4950'27,41"W W \\
\hline
\end{tabular}

\section{Amostragem}

Entre os meses de maio e junho de 2019, vinte amostras de água subterrânea foram coletadas nos pontos de amostragem distribuídos em nove bairros da Cidade de Parauapebas (Da Paz: 5 amostras; Beira Rio II: 4 amostras; Parque dos Carajás II: 3 amostras; Parque dos Carajás I: 2 amostras; Beira-Rio I: 2 amostras; Cidade Nova: 1 amostra; Nova Carajás: 1 amostra; Rio Verde: 1 amostra e União: 1 amostra).

As amostras foram coletadas em frascos de polietileno $(500 \mathrm{~mL})$ previamente esterilizados para realização das análises físico-químicas. Após a coleta, todas as amostras foram levadas para o Laboratório de Química do Campus de Parauapebas da Universidade Federal Rural da Amazônia devidamente identificadas, mantidas sobre refrigeração à temperatura de $4{ }^{\circ} \mathrm{C}$ e protegidas da luz para realização das análises físicoquímicas. Todos os parâmetros físico-químicos foram realizados em triplicata.

\section{Instrumentos e acessórios}

Um termômetro de mercúrio (marca Incoterm) com escala entre 0 e $50{ }^{\circ} \mathrm{C}$ foi utilizado para medir a temperatura no momento da coleta. As demais análises foram realizadas no laboratório, com phmetro de bancada (Marca Hanna Instruments, Modelo HI9321Q-799D), condutivímetro de bancada (Marca Hanna Instruments, Modelo HMCDB-150), turbidímetro de bancada (Marca Cheeselab, Modelo CL 1000). A cor, o teor de cloreto $(\mathrm{Cl})$ e os sólidos totais dissolvidos (STD) foram determinados de acordo com o Standard Methods for the Examination of Water and Wastewater from Americam Public Health Association (APHA, 2005).

Os materiais de plástico de polietileno, vidrarias e frascos volumétricos foram lavados com água corrente, depois com água desionizada e, em seguida foram imersos em banho em solução de $\mathrm{HNO}_{3} 10 \%$ $(\mathrm{v} / \mathrm{v})$ durante $24 \mathrm{~h}$. Posteriormente, esses materiais foram lavados abundantemente com água desionizada e secos a temperatura ambiente.

\section{Determinação dos parâmetros físico-químicos}

Foram analisados os seguintes parâmetros físico-químicos: A temperatura $(T)$, o potencial hidrogeniônico $(\mathrm{pH})$, a condutividade elétrica (CE) e a turbidez (TRB) foram medidas diretamente nas amostras brutas. A cor foi determinada pelo método de comparação visual (Método 2120B, APHA, 2005). 0 teor de cloreto $(\mathrm{Cl})$ foi determinado pelo método argentimétrico (Método 4500B) (APHA, 2005). Os sólidos totais dissolvidos (STD) foram determinados pelo método gravimétrico (Método 2540B) (APHA, 2005). Todas as análises foram realizadas em triplicata $(n=3)$, com exceção da temperatura somente uma replicata $(n=1)$. 
Aplicação estatística multivariada para a avaliação físico-química na qualidade da água subterrânea na cidade de Parauapebas (Sudeste do Estado do Pará)

CHAVES, H. S.; MORAIS, D. G.; DANTAS FILHO, H. A.; DANTAS, K. G. F.; BEIRAO, A. T. M.; SILVA, K. P.; SILVA, J. N.; SILVA, V. F. A.; SILVA, P. A.; CARVALHO, F. I. M.

\section{Tratamento estatístico dos dados}

Para o tratamento estatístico dos resultados, foram utilizados recursos básicos de estatística descritiva e métodos multivariados análise de componentes principais (PCA) e análise hierárquica de agrupamento (HCA), sendo processados com o auxílio do software Statistica 8.0 (StatSoft, Inc., Tulsa, OK, USA). O nível de significância obtido para a análise estatística dos dados foi de $p<0,05$.

Para realização da PCA e HCA, primeiramente construiu-se uma matriz de dados normalizadas contendo 20 linhas (amostras) e 7 colunas (variáveis: temperatura (T), pH, condutividade elétrica (CE), turbidez (TRB), cor, teor de cloreto $\left(\mathrm{Cl}^{-}\right)$, sólidos totais dissolvidos (STD)). O tipo de pré-processamento da matriz de dados originais foi o auto escalonamento, recurso utilizado em análise estatística onde se centra os dados na média e divide-se cada um pelo desvio padrão, de forma que todas variáveis passam a ter a mesma importância, ou seja, o mesmo peso, ocorrendo à normalização dos dados, procedimento que elimina a influência de diferentes unidades de medida (BEEBE et al., 1998; MINGOTI, 2005). Em seguida, os dados auto escalados foram submetidos à PCA e HCA para melhor interpretação das 20 amostras de águas subterrâneas.

A PCA foi utilizada neste estudo com o objetivo de reduzir a dimensionalidade de um conjunto de dados e detectar as variáveis mais significativas com a mínima perda dos dados originais, preservando ao mesmo tempo o máximo de informação (MINGOTI, 2005). Isto é feito através de cálculos de combinações lineares das variáveis originais, formando os componentes principais. Para isto, a matriz de dados originais é aproximada para duas matrizes menores (BEEBE et al., 1998). Desta forma, com o resultado da PCA foram obtidos gráficos bidimensionais de scores e loadings, que facilitam a visualização das informações dos dados experimentais, isto é, a distribuição das amostras e a importância das variáveis (CARVALHO et al., 2014).

Enquanto a HCA, por outro lado, é usada para analisar um conjunto de dados em termos de grupos definidos de maneira hierárquica, de acordo com a similaridade observada entre variáveis ou amostras, servindo de ferramenta complementar para PCA (MINGOTI, 2005). Neste estudo, a HCA foi realizada com base nos dados normalizados, utilizando o método Ward's, como método hierárquico aglomerativo e como medida de similaridade foi utilizada as distâncias Euclidianas normalizadas (BEEBE et al., 1998). Com base nos cálculos obteve-se o dendrograma das amostras de águas subterrâneas, o qual permite verificar o grau de similaridade entre os grupos formados (CARVALHO et., 2015).

\section{RESULTADOS E DISCUSSÃO}

\section{Avaliação dos parâmetros físico-químicos}

Os valores médios dos resultados obtidos correspondentes aos 7 parâmetros físico-químicos determinados nas amostras de águas subterrâneas estudadas estão apresentados na Tabela 2. Os resultados obtidos foram comparados com os valores padrões indicados pela legislação brasileira de referência, MS 2914/2011 e CONAMA 396/2008, acerca da qualidade de águas potável e subterrânea, respectivamente. 
Tabela 2: Valores obtidos para os parâmetros físico-químicos das amostras de águas subterrâneas.

\begin{tabular}{|c|c|c|c|c|c|c|c|c|c|c|c|c|c|c|c|c|c|c|c|c|c|}
\hline $\begin{array}{l}\text { Parâ } \\
\text { metro } \\
\text { s }\end{array}$ & $\begin{array}{l}\text { Unid } \\
\text { ades }\end{array}$ & $\begin{array}{l}\text { DP } \\
1\end{array}$ & $\begin{array}{l}\text { DP } \\
2\end{array}$ & $\begin{array}{l}\text { DP } \\
3\end{array}$ & $\begin{array}{l}\text { DP } \\
4\end{array}$ & $\begin{array}{l}\text { DP } \\
5\end{array}$ & $\begin{array}{l}\text { BR } \\
\text { II1 }\end{array}$ & $\begin{array}{l}\text { BR } \\
\text { II2 }\end{array}$ & $\begin{array}{l}\text { BR } \\
\text { II3 }\end{array}$ & $\begin{array}{l}\text { BR } \\
114\end{array}$ & $\begin{array}{l}\text { PC } \\
\text { II1 }\end{array}$ & $\begin{array}{l}\text { PC } \\
\text { II2 }\end{array}$ & $\begin{array}{l}\text { PC } \\
\text { II3 }\end{array}$ & $\begin{array}{l}\mathrm{P} \\
\mathrm{Cl} \\
1\end{array}$ & $\begin{array}{l}\mathrm{P} \\
\mathrm{Cl} \\
2 \\
\end{array}$ & $\begin{array}{l}\mathrm{B} \\
\mathrm{RI} \\
1 \\
\end{array}$ & $\begin{array}{l}\mathrm{B} \\
\mathrm{RI} \\
2 \\
\end{array}$ & $\begin{array}{l}C \\
N \\
1 \\
\end{array}$ & $\begin{array}{l}N \\
C \\
1 \\
\end{array}$ & $\begin{array}{l}\mathrm{R} \\
\mathrm{V} \\
1 \\
\end{array}$ & $\begin{array}{l}U \\
N \\
1\end{array}$ \\
\hline $\mathrm{T}$ & ${ }^{\circ} \mathrm{C}$ & $\begin{array}{l}27 \\
, 0\end{array}$ & $\begin{array}{l}26 \\
5\end{array}$ & $\begin{array}{l}27 \\
, 0\end{array}$ & $\begin{array}{l}26 \\
, 5\end{array}$ & $\begin{array}{l}25 \\
, 5\end{array}$ & $\begin{array}{l}26 \\
, 5\end{array}$ & $\begin{array}{l}26 \\
, 5\end{array}$ & $\begin{array}{l}27 \\
, 0\end{array}$ & $\begin{array}{l}27 \\
, 0\end{array}$ & $\begin{array}{l}26 \\
, 0\end{array}$ & $\begin{array}{l}26 \\
, 5\end{array}$ & $\begin{array}{l}25 \\
, 0\end{array}$ & $\begin{array}{l}2 \\
5 \\
5\end{array}$ & $\begin{array}{l}2 \\
6, \\
0\end{array}$ & $\begin{array}{l}27 \\
, 0\end{array}$ & $\begin{array}{l}27 \\
, 0\end{array}$ & $\begin{array}{l}2 \\
7, \\
0\end{array}$ & $\begin{array}{l}2 \\
6, \\
5\end{array}$ & $\begin{array}{l}2 \\
6, \\
0\end{array}$ & $\begin{array}{l}2 \\
7, \\
0\end{array}$ \\
\hline $\mathrm{pH}$ & - & $\begin{array}{l}5, \\
34\end{array}$ & $\begin{array}{l}5, \\
85\end{array}$ & $\begin{array}{l}5, \\
26\end{array}$ & $\begin{array}{l}5, \\
53\end{array}$ & $\begin{array}{l}5, \\
72\end{array}$ & $\begin{array}{l}5, \\
62\end{array}$ & $\begin{array}{l}6, \\
08\end{array}$ & $\begin{array}{l}5, \\
82\end{array}$ & $\begin{array}{l}5, \\
92\end{array}$ & $\begin{array}{l}5, \\
58\end{array}$ & $\begin{array}{l}5, \\
83\end{array}$ & $\begin{array}{l}6, \\
12\end{array}$ & $\begin{array}{l}6, \\
0 \\
5 \\
\end{array}$ & $\begin{array}{l}5, \\
6 \\
7 \\
\end{array}$ & $\begin{array}{l}5, \\
86\end{array}$ & $\begin{array}{l}5, \\
54\end{array}$ & $\begin{array}{l}5 \\
6 \\
0 \\
\end{array}$ & $\begin{array}{l}5, \\
4 \\
6\end{array}$ & $\begin{array}{l}5 \\
5 \\
7 \\
\end{array}$ & $\begin{array}{l}5, \\
6 \\
2 \\
\end{array}$ \\
\hline TRB & UNT & $\begin{array}{l}1, \\
5\end{array}$ & $\begin{array}{l}1, \\
5\end{array}$ & $\begin{array}{l}1, \\
25\end{array}$ & $\begin{array}{l}1 \\
75\end{array}$ & $\begin{array}{l}1, \\
0\end{array}$ & $\begin{array}{l}1, \\
75\end{array}$ & $\begin{array}{l}1, \\
5\end{array}$ & $\begin{array}{l}1, \\
25\end{array}$ & $\begin{array}{l}1, \\
25\end{array}$ & $\begin{array}{l}1, \\
0\end{array}$ & $\begin{array}{l}1, \\
25\end{array}$ & $\begin{array}{l}1, \\
5\end{array}$ & $\begin{array}{l}1, \\
5\end{array}$ & $\begin{array}{l}1, \\
5\end{array}$ & $\begin{array}{l}1, \\
5\end{array}$ & $\begin{array}{l}1, \\
0\end{array}$ & $\begin{array}{l}1, \\
2 \\
5\end{array}$ & $\begin{array}{l}1, \\
0\end{array}$ & $\begin{array}{l}1, \\
0\end{array}$ & $\begin{array}{l}1, \\
5\end{array}$ \\
\hline CE & $\begin{array}{l}\mu \mathrm{S} \\
\mathrm{cm}^{-1}\end{array}$ & $\begin{array}{l}16 \\
4, \\
4\end{array}$ & $\begin{array}{l}17 \\
6, \\
3\end{array}$ & $\begin{array}{l}13 \\
6, \\
3\end{array}$ & $\begin{array}{l}15 \\
8, \\
4\end{array}$ & $\begin{array}{l}21 \\
6, \\
7\end{array}$ & $\begin{array}{l}87 \\
, 2\end{array}$ & $\begin{array}{l}74 \\
, 0\end{array}$ & $\begin{array}{l}72 \\
, 2\end{array}$ & $\begin{array}{l}65 \\
, 5\end{array}$ & $\begin{array}{l}68 \\
, 3\end{array}$ & $\begin{array}{l}74 \\
1\end{array}$ & $\begin{array}{l}60 \\
, 2\end{array}$ & $\begin{array}{l}7 \\
7, \\
3\end{array}$ & $\begin{array}{l}5 \\
9, \\
4\end{array}$ & $\begin{array}{l}71 \\
, 7\end{array}$ & $\begin{array}{l}55 \\
, 0\end{array}$ & $\begin{array}{l}5 \\
4, \\
3 \\
\end{array}$ & $\begin{array}{l}8 \\
1, \\
6\end{array}$ & $\begin{array}{l}5 \\
1, \\
0\end{array}$ & $\begin{array}{l}6 \\
3, \\
8\end{array}$ \\
\hline Cor & $\mathrm{uH}$ & $\begin{array}{l}10 \\
, 0\end{array}$ & $\begin{array}{l}5, \\
0\end{array}$ & $\begin{array}{l}5, \\
0\end{array}$ & $\begin{array}{l}15 \\
, 0\end{array}$ & $\begin{array}{l}15 \\
, 0\end{array}$ & $\begin{array}{l}5, \\
0\end{array}$ & $\begin{array}{l}10 \\
, 0\end{array}$ & $\begin{array}{l}7, \\
5\end{array}$ & $\begin{array}{l}10 \\
, 0\end{array}$ & $\begin{array}{l}10 \\
, 0\end{array}$ & $\begin{array}{l}10 \\
, 0\end{array}$ & $\begin{array}{l}5, \\
0\end{array}$ & $\begin{array}{l}5, \\
0\end{array}$ & $\begin{array}{l}5, \\
0\end{array}$ & $\begin{array}{l}10 \\
, 0\end{array}$ & $\begin{array}{l}10 \\
, 0\end{array}$ & $\begin{array}{l}7, \\
5\end{array}$ & $\begin{array}{l}7, \\
5\end{array}$ & $\begin{array}{l}1 \\
0, \\
0\end{array}$ & $\begin{array}{l}1 \\
0, \\
0\end{array}$ \\
\hline $\mathrm{Cl}^{-}$ & $\begin{array}{l}\mathrm{mg} \mathrm{L}^{-} \\
\end{array}$ & $\begin{array}{l}21 \\
, 0\end{array}$ & $\begin{array}{l}17 \\
, 4\end{array}$ & $\begin{array}{l}20 \\
, 4\end{array}$ & $\begin{array}{l}12 \\
, 7\end{array}$ & $\begin{array}{l}26 \\
, 1\end{array}$ & $\begin{array}{l}23 \\
, 2\end{array}$ & $\begin{array}{l}10 \\
6\end{array}$ & $\begin{array}{l}15 \\
, 3\end{array}$ & $\begin{array}{l}11 \\
8\end{array}$ & $\begin{array}{l}14 \\
1\end{array}$ & $\begin{array}{l}11 \\
, 0\end{array}$ & $\begin{array}{l}18 \\
, 3\end{array}$ & $\begin{array}{l}1 \\
5, \\
5\end{array}$ & $\begin{array}{l}1 \\
2, \\
7\end{array}$ & $\begin{array}{l}12 \\
, 2\end{array}$ & $\begin{array}{l}10 \\
, 2\end{array}$ & $\begin{array}{l}1 \\
4, \\
7\end{array}$ & $\begin{array}{l}1 \\
6, \\
2\end{array}$ & $\begin{array}{l}2 \\
5, \\
7\end{array}$ & $\begin{array}{l}1 \\
6, \\
4\end{array}$ \\
\hline STD & ${ }_{1} \mathrm{mg} \mathrm{L}^{-}$ & $\begin{array}{l}17 \\
7 \\
5\end{array}$ & $\begin{array}{l}20 \\
3, \\
0\end{array}$ & $\begin{array}{l}18 \\
5 \\
5\end{array}$ & $\begin{array}{l}17 \\
5, \\
0\end{array}$ & $\begin{array}{l}27 \\
1, \\
8\end{array}$ & $\begin{array}{l}91 \\
, 5\end{array}$ & $\begin{array}{l}83 \\
, 0\end{array}$ & $\begin{array}{l}87 \\
, 0\end{array}$ & $\begin{array}{l}74 \\
, 0\end{array}$ & $\begin{array}{l}76 \\
, 0\end{array}$ & $\begin{array}{l}85 \\
, 5\end{array}$ & $\begin{array}{c}70 \\
, 5\end{array}$ & $\begin{array}{l}8 \\
8, \\
5\end{array}$ & $\begin{array}{l}6 \\
9, \\
3\end{array}$ & $\begin{array}{l}77 \\
, 5\end{array}$ & $\begin{array}{l}71 \\
, 5\end{array}$ & $\begin{array}{l}6 \\
8, \\
0\end{array}$ & $\begin{array}{l}7 \\
9, \\
0\end{array}$ & $\begin{array}{l}6 \\
4, \\
8\end{array}$ & $\begin{array}{l}5 \\
6, \\
5\end{array}$ \\
\hline
\end{tabular}

Os valores de estatística descritiva e os valores máximos permitidos (VMP) para os parâmetros físicoquímicos determinados nas amostras de águas subterrâneas estão apresentados na Tabela 3.

Tabela 3: Valores de estatística descritiva e VMP para todos os parâmetros físico-químicos determinados nas amostras de água subterrânea $(N=20)$.

\begin{tabular}{l|l|l|l|l|l}
\hline Parâmetros & Unidades & Média \pm SD & Mínimo - Máximo & MS 2914/2011 ${ }^{\text {a }}$ & CONAMA 396/2008 $^{\text {a }}$ \\
\hline \multirow{2}{*}{$\mathrm{T}$} & ${ }^{\circ} \mathrm{C}$ & $26,45 \pm 0,46$ & $25,0-27,0$ & - & - \\
\cline { 2 - 6 } & - & $5,70 \pm 0,82$ & $5,26-6,12$ & 6,0 a 9,51- & - \\
\hline TRB & UNT & $1,33 \pm 0,36$ & $1,0-1,75$ & 5 & - \\
\hline CE & $\mu S$ cm$^{-1}$ & $93,38 \pm 45,2$ & $51,0-216,7$ & - & - \\
\hline Cor & $\mathrm{uH}$ & $8,62 \pm 2,76$ & $5,0-15,0$ & 15 & - \\
\hline $\mathrm{Cl}$ & $\mathrm{mg} \mathrm{L}^{-1}$ & $16,27 \pm 6,35$ & $10,2-26,1$ & 250 & 250 \\
\hline STD & $\mathrm{mg} \mathrm{L}^{-1}$ & $107,80 \pm 64,6$ & $56,5-271,8$ & 1000 & 1000 \\
\hline
\end{tabular}

$\mathrm{SD}=$ desvio-padrão; ${ }^{a}$ valores máximos permitidos (VMP) e ${ }^{1}$ faixa recomendada pela Portaria № 2914/2011 do MS.

Os valores obtidos de temperatura em todas as amostras variaram de 25 a $27^{\circ} \mathrm{C}$, apresentando uma amplitude térmica pequena, isto é, as temperaturas nas águas não sofreram grandes mudanças (DIAS et al., 2004). O valor de pH nas amostras variou de 5,26 a 6,12, observando-se que na área de estudo temos águas com características predominantemente ácidas. Somente as amostras BRII2, PCl1 e PCII3 obtiveram pH de acordo com o valor recomendado pelo MS 2914/2011 (6,0 a 9,5), enquanto as demais amostras o pH variou de 5,26 a 5,92, sendo consideradas águas levemente ácidas, refletindo a acidez característica das águas subterrâneas da região (AZEVEDO, 2006; CABRAL et al., 2006). De acordo com Carvalho et al. (2015), águas subterrâneas que apresentam pH ácido pode ser indício de contaminação dos poços tubulares por fossas e esgotos domésticos. Vale ressaltar que o pH das águas naturais influencia na solubilidade de compostos contendo metais traços, com a ordem decrescente de solubilidade, $\mathrm{Cd}>\mathrm{Cu}>\mathrm{Pb}$, sendo esta fração solúvel e biodisponível para interagir com organismos vivos (FONTENELLE et al., 2009).

Os valores de condutividade elétrica variaram de 51,0 a $216,7 \mu \mathrm{S} \mathrm{cm}^{-1}$ e encontram-se abaixo dos 
Aplicação estatística multivariada para a avaliação físico-química na qualidade da água subterrânea na cidade de Parauapebas (Sudeste do Estado do Pará)

CHAVES, H. S.; MORAIS, D. G.; DANTAS FILHO, H. A.; DANTAS, K. G. F.; BEIRAO, A. T. M.; SILVA, K. P.; SILVA, J. N.; SILVA, V. F. A.; SILVA, P. A.;

teores obtidos em estudos de outros autores (CABRAL et al., 2006; CARVALHO et al., 2015). Todas as amostras de água subterrâneas coletadas no bairro Da Paz apresentaram CE $>100 \mu \mathrm{S} \mathrm{cm}^{-1}$, indicando altos teores de sais dissolvidos ionizados, podendo as mesmas estarem contaminadas decorrentes do despejo de efluentes domésticos, com isso aumentando o grau de salinidade destas águas (SANTOS et., 2011).

Os valores obtidos de teor de cloreto $\left(10,2\right.$ a $\left.25,7 \mathrm{mg} \mathrm{L}^{-1}\right)$, sólidos totais dissolvidos (56,5 a 271,8 mg $\mathrm{L}^{-1}$ ) e turbidez (1,0 a 1,75 NTU), encontram-se abaixo dos valores máximos permitidos pela MS 2914/2011 e CONAMA 396/2008. Porém, as amostras com teores mais elevados de sólidos totais dissolvidos e condutividade elétrica estão fortemente associadas à contaminação pela descarga de efluentes domésticos (SANTOS et al., 2011). A cor variou de 5,0 a 15,0 $\mu \mathrm{H}$, mas somente as amostras DP4 e DP5 coletadas no bairro Da Paz apresentaram valores iguais ao limite máximo permitido $(15,0 \mu \mathrm{H})$ pela MS $2914 / 2011$. Isto ocorre porque estas amostras sofrem influência da maior carga de material sólido em suspensão (BAIG et al., 2009).

\section{Métodos multivariados}

Para melhor extração de informações das amostras e variáveis e uma avaliação mais minuciosa dos dados, aplicou-se a análise de componentes principais (PCA) e análise hierárquica de agrupamento (HCA) a fim de conduzir a uma melhor interpretação dos dados multivariados e observar semelhanças e/ou diferenças entre as amostras de aguas subterrâneas, a partir de suas características físico-químicas apresentadas na Tabela 3.

Desta forma, a PCA projetou os dados em um espaço com 7 dimensões no plano (7 PCs), sendo que de acordo com o critério de Kaiser, somente as duas componentes principais iniciais (PC1: 46,8\% e PC2: $28,7 \%$ ) foram consideradas as mais significativas para variância explicada dos dados, pois cada componente apresentou autovalores > 1 (CARVALHO et al., 2015; ALEX et al., 2019; ALVES et al., 2019), totalizando 75,5\% da variância total dos dados originais. Os pesos dos coeficientes referentes aos loadings para as duas componentes principais mais significativas com seus respectivos autovalores, variância explicada e variância acumulada estão apresentados na Tabela 4.

Tabela 4: Loadings para as componentes principais, autovalores e variância total (\%) para matriz de $20 \times 7$. Os valores mais significativos estão em negrito.

\begin{tabular}{lll}
\hline Componentes & PC1 & PC2 \\
\hline T & 0,076 & $\mathbf{0 , 8 1 5}$ \\
pH & $\mathbf{0 , 6 6 4}$ \\
TRB & $\mathbf{0 , 4 7 0}$ & $-0,405$ \\
CE & 0,086 & $-0,135$ \\
Cor & $-\mathbf{0 , 9 3 2}$ & 0,371 \\
Cl & $-\mathbf{0 , 4 1 1}$ & $-0,283$ \\
STD & $-\mathbf{0 , 6 5 1}$ & 0,134 \\
Autovalores & $-\mathbf{0 , 9 3 7}$ & 1,52 \\
Variância explicada (\%) & 2,57 & 28,70 \\
Variância acumulada (\%) & 46,80 & $\mathbf{7 5 , 5 0}$ \\
\hline
\end{tabular}

Na Figura 3, através da projeção PC1 x PC2, observou-se nitidamente no gráfico dos scores obtido a separação de dois grupos (destacados em círculos), denominados de grupos A e B. Os mesmos caracterizam 
uma diferença entre as amostras de águas subterrâneas coletadas nos bairros da Beira Rio I, Beira Rio II, Parque dos Carajás I, Parque dos Carajás II, Nova Carajás, Cidade Nova, Rio Verde e União (grupo A, total de 15 amostras) com as coletadas no bairro Da Paz (grupo B, total de 5 amostras). Esta separação entre as amostras ocorreu por causa da influência de suas características físico-químicas distintas.

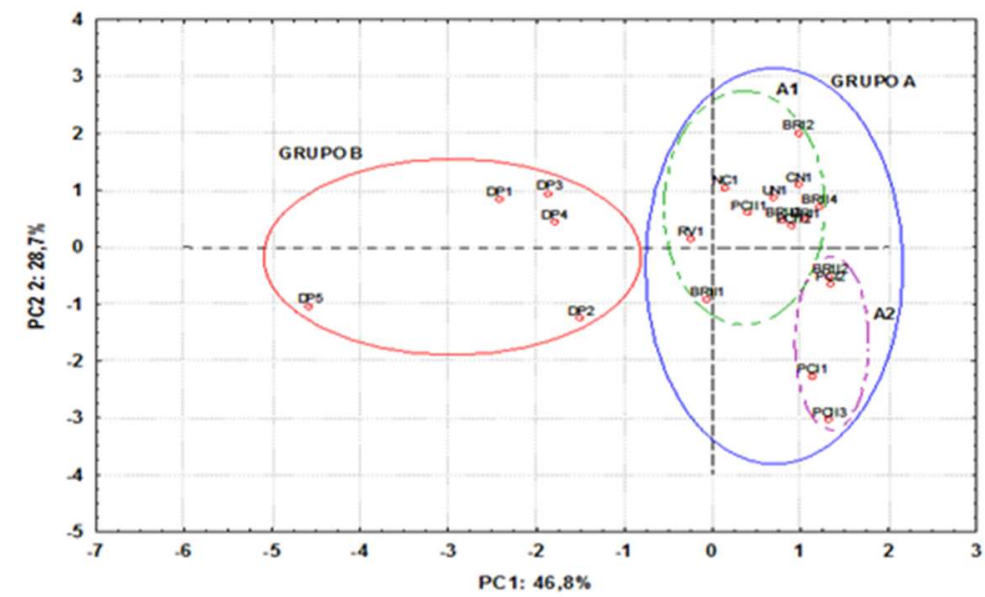

Figura 3: Gráfico dos scores PC1 x PC2 correspondentes às amostras de águas subterrâneas estudadas.

Na Figura 4, pode ser observado no gráfico dos loadings, através da projeção da PC1 x PC2, quais as variáveis que mais contribuíram para a discriminação das amostras.

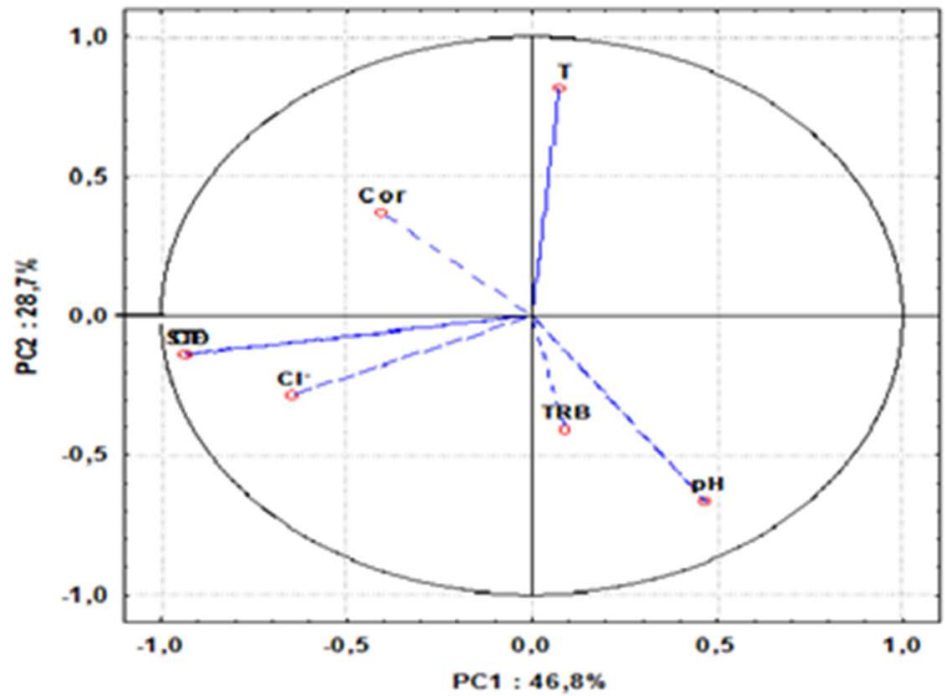

Figura 4: Gráfico dos loadings PC1 x PC2 correspondentes aos parâmetros físico-químicos determinados nas amostras de águas subterrâneas.

A primeira componente principal (PC1) foi a que mais contribuiu para a separação das amostras, explicando $46,8 \%$ da variância dos dados, concentrando informações a respeito das variáveis CE, STD, Cor e

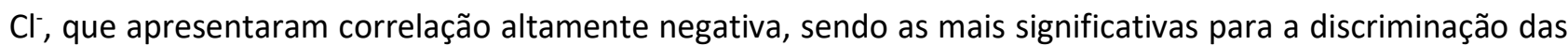
amostras do grupo B (DP5, DP2, DP4, DP3 e DP1) em relação às amostras do grupo A (BRI1, BRI2, BRII1, BRII2, BRII3, BRII4, PCI1, PCI2, PCII1, PC2II, PCII3, CN1, NC1, RV1 e UN1), consequência dos valores mais elevados de CE, STD, Cor e $\mathrm{Cl}^{-}$para amostras contidas no grupo B (Tabela 2). Por outro lado, a T, TRB e pH estão correlacionadas positivamente na PC1, exercendo certa influência na formação das amostras presentes no 
Aplicação estatística multivariada para a avaliação físico-química na qualidade da água subterrânea na cidade de Parauapebas (Sudeste do Estado do Pará)

CHAVES, H. S.; MORAIS, D. G.; DANTAS FILHO, H. A.; DANTAS, K. G. F.; BEIRAO, A. T. M.; SILVA, K. P.; SILVA, J. N.; SILVA, V. F. A.; SILVA, P. A.;

grupo A.

Os resultados obtidos das associações entre as variáveis físico-químicas e amostras observados através da projeção bidimensional das PCs (PC1 x PC2) com maior variância explicada $(75,5 \%)$ foram confirmados pelos agrupamentos formados na HCA. No dendrograma apresentado na Figura 5, as linhas verticais representam as amostras e as linhas horizontais representam as medidas de similaridades calculadas empregando-se as distâncias Euclidianas, as quais foram responsáveis para formação de agrupamentos entre as amostras de águas subterrâneas em termos de similaridades de acordo os dados normalizados.

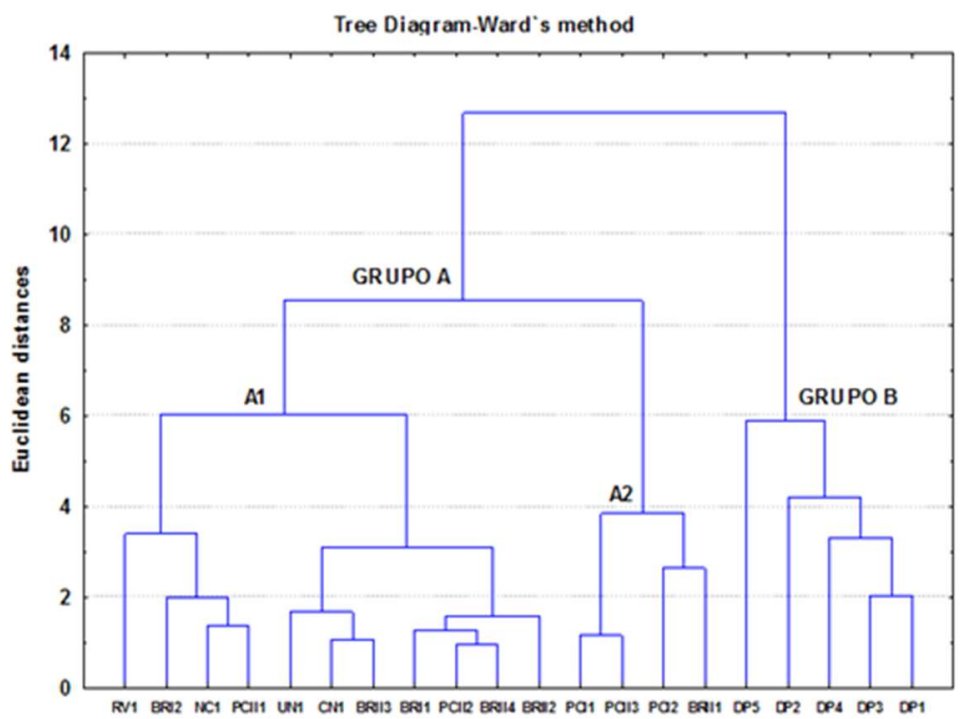

Figura 5: Dendrograma obtido para as amostras de águas subterrâneas a partir das distâncias Euclidianas normalizadas.

Inicialmente observar-se claramente no dendrograma apresentado na Figura 5, a formação de dois agrupamentos separados pela máxima dissimilaridade, identificados como agrupamentos A e B com distância Euclidiana de 12,8. Analisando o dendrograma no sentido da esquerda para direita, observa-se que o agrupamento A apresentou distância Euclidiana 8,6. Este agrupamento foi formado pelo subagrupamento A1 (amostras RV1, BRI2, NC1, PCII1, UN1, CN1, BRII3, BRI1, PC2II, BRII4 e BRII2) e subagrupamento A2 (amostras PCI1, PCII3, PCI2 e BRII1), demonstraram menor similaridade de acordo com a análise dos subagrupamentos formados na HCA, corroborando com as interpretações feitas na PCA no gráfico dos scores (Figura 3).

O agrupamento B apresentou distância Euclidiana 6,0. Este agrupamento foi formado pelas amostras DP5, DP2, DP4, DP3 e DP1, respectivamente, demonstrando maior similaridade quando comparado com o agrupamento A, agregando com as observações realizadas no gráfico dos scores da PC1 x PC2.

\section{CONCLUSÕES}

Os resultados obtidos para todos os parâmetros físico-químicos estudados mostraram que todas as amostras de águas subterrâneas atenderam os limites estabelecidos pela Portaria MS 2914/2011 e Resolução CONAMA 396/2008, não caracterizando um potencial risco para a saúde da população dos bairros da Cidade 
Aplicação estatística multivariada para a avaliação físico-química na qualidade da água subterrânea na cidade de Parauapebas (Sudeste do Estado do Pará)

CHAVES, H. S.; MORAIS, D. G.; DANTAS FILHO, H. A.; DANTAS, K. G. F.; BEIRAO, A. T. M.; SILVA, K. P.; SILVA, J. N.; SILVA, V. F. A.; SILVA, P. A.;

de Parauapebas onde foram realizadas coletas de águas subterrâneas que fazem uso de poços tubulares para captação de águas subterrâneas para consumo humano. Porém, após a avaliação dos resultados mais elevados de condutividade elétrica, sólidos totais dissolvidos e cor, para as amostras de águas subterrâneas coletadas no bairro Da Paz, servindo de indicativos para identificação de possíveis atividades antropogênicas de contaminação, principalmente em relação à descarga de efluentes domésticos. Com o auxílio de métodos multivariados, a PCA e HCA aplicada nos dados obtidos dos parâmetros físico-químicos determinados nas 20 amostras de águas subterrâneas, revelaram diferenças significativas entre as amostras estudadas, sendo possível observar a formação de dois grupos distintos (A e B).

Os parâmetros físico-químicos mais significativos para a separação dos dois grupos foram temperatura, $\mathrm{pH}$, condutividade elétrica, cor, teor de cloreto, sólidos totais dissolvidos. Assim, a análise estatística multivariada dos dados obtidos pela PCA e HCA serviu como uma ferramenta exploratória na análise e interpretação dos dados originais. Foi possível extrair informações relevantes e capazes de identificar semelhanças e diferenças apresentadas pelas amostras de águas subterrâneas, correlacionandoas com os parâmetros físico-químicos estudados.

AGRADECIMENTOS: Os autores agradecem ao Conselho Nacional de Desenvolvimento Científico e Tecnológico (CNPq, Brasília, Brasil) pela bolsa concedida de iniciação científica e voluntariado aos discentes, Henrique Souza Chaves e Denilton Galvão de Morais, respectivamente (Edital PROPED no 04/2018).

\section{REFERÊNCIAS}

ALVES, B. S. F.; JUNIOR, J. B. P.; CARVALHO, F. I. M.; FILHO, H. A. D.; FERNANDES DANTAS, KELLY G.. Mineral Composition of Amazonian Fruits by Flame Atomic Absorption Spectrometry Using Multivariate Analysis. Biological Trace Element Research, v.189, p.259-266, 2019. DOI: https://doi.org/10.1007/s12011-018-1451-6

APHA. American Public Health Association. Standard Methods for the Examination of Water and Wastewater. 21 ed. Washington: APHA, 2005.

AZEVEDO, R. P.. Uso de água subterrânea em sistema de abastecimento público de comunidades na várzea da Amazônia central. Acta Amazônica, v.36, n. 3, p.312-320, 2006. DOI: https://doi.org/10.1590/S0044$\underline{59672006000300004}$

BAIG, J. A.; KAZI, T. G.; ARAIN, M. B.; AFRIDI, H. I.; KANDHRO G. A.; SARFRAZ, R. A.; JAMAL, M. K.; SHAH, A. Q.. Evaluation of arsenic and other physicochemical parameters of surface and groundwater of Jamshoro, Pakistan. Journal of Hazardous Materials, v.166, p.662-669, 2009. DOI: https://doi.org/10.1016/j.jhazmat.2008.11.069

BEEBE, K. R.; PELL, R. J.; SEASHOLTZ, M. B.. Chemometrics: A Pratical Guide, John Wiley \& Sons: New York, 1998.

BRASIL. Ministério da Saúde. Portaria n. 2914 de 12 de dezembro de 2011. Brasília: DOU, 2011.
CABRAL, N. M. T.. Teores de (NO3-) e amônio ( $\left.\mathrm{NH}^{+}\right)$nas águas do aquífero Barreiras nos bairros do Reduto, Nazaré e Umarizal - Belém/PA. Química Nova, v.30, p.1804-1808, 2007. DOI: https://doi.org/10.1590/S010040422007000800003

CABRAL, N. M. T.; LIMA, L. M.. Comportamento hidrogeoqímico das águas do aquífero Barreiras nos bairros centrais de Belém, Pará. Boletim do Museu Paraense Emílio Goeldi. Ciências Naturais, v.1, n. 1, p.149-166, 2006.

CAMPANHA, M. B.; MELO, C. A.; MOREIRA, A. B.; FERRARESE, R. F. M. S.; TADINI, A. M.; GARBIN, E. V.; BISINOTI, M. C.. Variabilidade espacial e temporal de parâmetros físico-químicos nos rios turvo, preto e grande no Estado de São Paulo, Brasil. Química Nova, v.33, n. 9, p.1831-1836, 2010. DOI: https://doi.org/10.1590/S0100$\underline{40422010000900002}$

CARVALHO, F. I. M.; DANTAS FILHO, H. A.; DANTAS, K. G. F.. Simultaneous determination of 16 polycyclic aromatic hydrocarbons in groundwater by GC-FID after solid-phase extraction. SN Applied Sciences, v.1, n.804, 2019. DOI: https://doi.org/10.1007/s42452-019-0839-z

CARVALHO, F. I. M.; LEMOS, V. P.; DANTAS FILHO, H. A.; DANTAS, K. G. F.. Assessment of Groundwater Quality from the Belém Based on Physicochemical Parameters and Levels 
Aplicação estatística multivariada para a avaliação físico-química na qualidade da água subterrânea na cidade de Parauapebas (Sudeste do Estado do Pará)

CHAVES, H. S.; MORAIS, D. G.; DANTAS FILHO, H. A.; DANTAS, K. G. F.; BEIRAO, A. T. M.; SILVA, K. P.; SILVA, J. N.; SILVA, V. F. A.; SILVA, P. A.;

of Trace Elements Using Multivariate Analysis. Revista Virtual de Química, v.7, n.6, p.2221-2241, 2015. DOI: https://doi.org/10.5935/1984-6835.20150132

CHAVES, H. S.; MORAIS, D. G.; COSTA, K. A. D.; DANTAS, K. G. F.; SILVA, C. R.; SILVA, J. N.; SILVA, J. P.; SILVA, V. F. A.; SILVA, P. A.; CARVALHO, F. I. M.. Estudo da qualidade das águas subterrâneas de abastecimento em bairros na cidade de parauapebas a partir de parâmetros físico-químicos. Revista Ibero-americana de Ciências Ambientais, v.11, p.113-121, 2020. DOI: https://doi.org/10.6008/CBPC2179$\underline{6858.2020 .003 .0011}$

CONAMA. Conselho Nacional de Meio Ambiente. Resolução n. 396 de 3 de abril de 2008. Brasília: DOU, 2008.

CONCEIÇÃO, F. T.; SARDINHA, D. S.; SOUZA, A. D. G.; NAVARRO, G. R. B.. Anthropogenic influences on annual flux of cations and anions at meio stream basin, São Paulo State, Brasil. Water, Air, and Soil Pollution, n.205, p.79-91, 2010. DOI: https://doi.org/10.1007/s11270-009-0057-1

DIAS, J. C.; LIMA, W. N.. Comparação de métodos para a determinação de matéria orgânica em amostras ambientais. Revista Científica da UFPA, v.4, p.1-16, 2004.

DÓREA, H. S.; BISPO, J. R. L.; ARAGÃO, K. A. S.; CUNHA, B. B.; NAVICKIENE, S.; ALVES, J. P. H.; ROMÃO, L. P. C.; GARCIA, C. A. B.. Analysis of BTEX, PAHs and metals in the oilfield produced water in the State of Sergipe, Brazil. Microchemical Journal, v.85, p.234-238, 2007. DOI: https://doi.org/10.1016/j.microc.2006.06.002

FONTENELE, A. P. G.; PEDROTTI, J. J.; FORNARO, A.. Avaliação de metais traços e íons majoritários em águas subterrâneas na cidade de São Paulo. Química Nova, v.32, n.4, p.839-844, 2009. DOI: https://doi.org/10.1590/S0100$\underline{40422009000400003}$

FORTE, E. J.; AZEVEDO, M. S.; OLIVEIRA, R. C.; ALMEIDA, R.. Contaminação de aquífero por hidrocarbonetos: Estudo de caso na Vila Tupi, Porto Velho - Rondônia. Química Nova, v.30, n.7, p.1539-1544, 2007. DOI: https://doi.org/10.1590/S0100-40422007000700008

MARTINS, A. S.; JUNIOR, J. B. P.; GOMES, A. A.; CARVALHO, F. I. M.; FILHO, H. A. D.; DAS GRAÇAS FERNANDES DANTAS, K. G. F.. Mineral Composition Evaluation in Energy Drinks Using ICP OES and Chemometric Tools. Biological Trace Element Research, v.194, p.284-294, 2019. DOI: https://doi.org/10.1007/s12011-019-01770-y

MINGOTI, S. A.. Análise de dados através de métodos de estatística multivariada. Belo Horizonte: UFMG, 2005.

PANERO, F. S.; SILVA, H. E. B.. Application of exploratory data analysis for the characterization of tubular wells of the North of Brazil. Microchemical Journal, v.88, p.194-200, 2008. DOI: https://doi.org/10.1016/j.microc.2007.11.020

REBOUÇAS, A. C.; BRAGA, B.; TUNDISI, J. G.. Águas doces no Brasil: capital ecológico, uso e conservação. 3 ed. São Paulo: Escrituras, 2006.

SANTOS, J. S.; SANTOS, M. L. P.; ALEXANDRINO, D. M.. Comparative study of the salinalization process in surface water reservoirs located in two distinct regions in southwestern Bahia, Brazil. Journal of the Brazilian Chemical Society, v.22, n.8, p.1418-1425, 2011. DOI: https://doi.org/10.1590/S0103-50532011000800004

A CBPC - Companhia Brasileira de Produção Científica (CNPJ: 11.221.422/0001-03) detém os direitos materiais desta publicação. Os direitos referem-se à publicação do trabalho em qualquer parte do mundo, incluindo os direitos às renovações, expansões e disseminações da contribuição, bem como outros direitos subsidiários. Todos os trabalhos publicados eletronicamente poderão posteriormente ser publicados em coletâneas impressas sob coordenação da Sustenere Publishing, da Companhia Brasileira de Produção Científica e seus parceiros autorizados. Os (as) autores (as) preservam os direitos autorais, mas não têm permissão para a publicação da contribuição em outro meio, impresso ou digital, em português ou em tradução. 